\title{
Gestion d'un édentement complet maxillaire présentant une crête flottante antérieure
}

\section{RÉSUMÉ}

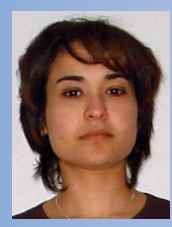

Jihane SLAOUI HASNAOUI

Résidente au Service de prothèse adjointe Centre de traitement

et de consultation dentaire de Rabat.

38 avenue Abtal,

rue Oum Rabiae, appt 8 Agdal,

Rabat, Maroc.

Amal SEFRIOUI

Professeur assistant

au Département de prothèse adjointe

de la muqueuse buccale,

Faculté de médecine dentaire de Rabat

Centre de traitement

et de consultation dentaire de Rabat.

\section{Olivier FROMENTIN}

MCU-PH au Département de prothèse

Service d'Odontologie,

Hôpital Hôtel-Dieu 1.

U.F.R. d'Odontologie,

Université Denis-Diderot,

Paris 7.

\section{Ahmed ABDEDINE}

Professeur de l'enseignement supérieur Chef de service de prothèse adjointe, Faculté de médecine dentaire de Rabat, Centre de traitement

et de consultation dentaire de Rabat. Rabat, Maroc
Les crêtes flottantes sont présentes dans $24 \%$ des édentements maxillaires avec une prédilection pour les régions antérieures. Elles sont souvent associées au syndrome décrit par Kelly.

Sur le plan histologique, la crête flottante présente un caractère irréversible. De ce fait, la mise en condition tissulaire ne peut apporter les améliorations escomptées.

Les trois principales approches thérapeutiques curatives pour gérer cette situation clinique sont :

- I'exérèse chirurgicale de la crête flottante et la restauration de l'arcade maxillaire par prothèse amovible conventionnelle ;

- la réalisation de prothèse complète supra-implantaire maxillaire associée ou non aux greffes osseuses ;

- la réalisation de la prothèse amovible complète conventionnelle avec des techniques d'empreintes spécifiques.

Le but de cet article est de faire la synthèse des différentes possibilités thérapeutiques disponibles et de discuter leurs indications. crêtes flottantes

- édentement maxillaire

- prothèses amovibles complètes

- résorption maxillaire 


\section{Introduction}

$>$

Chez l'édenté total, l'intégrité de la surface d'appui ostéomuqueuse est un facteur important pour l'équilibre de la future prothèse amovible.

Les tissus de soutien subissent l'influence de plusieurs facteurs généraux et locaux. La résorption osseuse physiologique, la sénescence ainsi que les remaniements engendrés par les maladies systémiques et la polymédication entrainent des modifications de ces tissus. De même, les traumatismes engendrés par une prothèse mal réalisée ou mal adaptée parceque trop ancienne influencent le degré de résorption osseuse [1] et le développement d'hyperplasies tissulaires.

L'étiologie de la résorption osseuse est multifactorielles, complexe, avec de fortes variations individuelles qui sont souvent inexpliquées. Même si aucune étude n'a pu établir l'importance des différents facteurs les uns par rapport aux autres, il est souvent rapporté que la pression mécanique excessive chronique en rapport avec les contraintes occlusales est responsable de l'apparition de zones de résorption locales [2].
Les crêtes flottantes sont composées d'un tissu fibreux épais, non adhérent aux plans profonds. Elles sont situées sur le sommet des crêtes édentées et compensent le volume perdu lors de la fonte osseuse [3]. L'examen au microscope du tissu de la crête flottante révèle des connexions fibreuses denses et matures, des paquets de fibres de collagène avec peu d'éléments cellulaires, de rares cellules inflammatoires et un tissu épithélial pseudo normal avec quelques signes d'hyperplasie [4]. Des traces de cartilage métaplasique et/ou de tissu osseux peuvent y être également repérées [5]. La rupture des insertions fibrillaires à l'os sousjacent ne permet plus à la fibromuqueuse de transmettre la charge occlusale aux tissus calcifiés, et entraîne leur résorption inéluctable [6].

Quels sont les facteurs favorisant l'apparition des crêtes flottantes maxillaires antérieures? Et quelles sont les différentes possibilités thérapeutiques disponibles pour gérer un édentement maxillaire complet présentant cette hyperplasie antérieure?

\section{Etiopathogénie des crêtes flottantes maxillaires antérieures}

Les crêtes flottantes sont présentes dans $24 \%$ des édentements maxillaires avec une prédilection pour les régions antérieures (la zone la plus faible de l'arcade maxillaire en termes de résistance aux contraintes fonctionnelles [7]), alors que leur prévalence n'est que de $5 \%$ à la mandibule [8-10].
Elles sont souvent associées au syndrome décrit par Kelly, en 1972, dénommé «Syndrome combiné " ou encore "Syndrome d'hyperfonction antérieure » [11]. Ce syndrome a été décrit dans le cadre d'un édentement complet maxillaire restauré par une prothèse amovible ancienne 
associée à un édentement mandibulaire bilatéral postérieur non traité.

Pour Kelly [12], le facteur déclenchant des signes de ce syndrôme serait la résorption prématurée de la crête antérieure maxillaire. Saunders et al. [13] ont suggéré que ce syndrome serait plutôt initié par la perte de calage et de contacts équilibrants postérieurs. Ceuxci seraient responsables de l'apparition d'un trauma occlusal antéro-supérieur du fait de I'action traumatique du bloc incisivo-canin inférieur entrainant une résorption progressive de la crête osseuse maxillaire sous prothétique. Cette résorption serait compensée par l'apparition d'une fibromatose réactionnelle.

En fait, Palmqvist et al. [14] ont constaté que : - il n'existait pas d'études épidémiologiques concernant le syndrome combiné ;

- il n'existait pas d'études longitudinales étudiant la résorption maxillaire avec extractions des dents antérieures comme point de départ ;

- les études comportaient des échantillons très réduits de patients.

Ces auteurs ont rapporté qu'il n'existait pas de lien clair entre la résorption au niveau des crêtes maxillaires antérieures et la présence de dents antérieures mandibulaires. De même, il n'existe pas de preuve scientifique confirmant que la restauration de l'édentement terminal mandibulaire par une prothèse amovible partielle préviendrait le développement au maxillaire d'une crête flottante antérieure.

De même, dans le traitement de l'édentement complet par PAT (prothèse amovible totale) maxillaire face à une prothèse supra implantaire mandibulaire, l'apparition de résorption osseuse antérieure maxillaire a été rapportée.
Ce phénomène se produirait lors de la réalisation d'une PACSI (prothèse amovible complète supra-implantaire) stabilisée par barre de conjonction [15] et dans une moindre mesure, lors de I'utilisation d'attachements axiaux magnétiques [16].

L'étude rétrospective de Kreisler et al. [17] d'une durée de huit années a montré que les prothèses amovibles complètes supra-implantaires avec deux implants symphysaires connectés à une barre de conjonction semblent entrainer une résorption maxillaire plus importante au niveau antérieur qu'au niveau des secteurs postérieurs.

Cela semble en accord avec l'étude récente de Nârhi et al. [15], qui montre que la résorption horizontale de la crête maxillaire serait indépendante du type de restauration mandibulaire.

En revanche, pour Gupta et al. [18], la prothèse complète fixée supra-implantaire mandibulaire ne semblerait pas promouvoir les symptômes décrits par Kelly, même si la perte de contacts postérieurs a été rapportée.

A la lumière de ces données, les variations individuelles, la fragilité de la crête antérieure maxillaire et la surcharge occlusale antérieure induite par la perte des contacts occlusaux postérieurs à long terme, sont les facteurs dominants dans l'apparition de la crête flottante antérieure.

Une arcade mandibulaire avec des dents antérieures naturelles ou une arcade mandibulaire restaurée par prothèse amovible complète supra-implantaire avec une barre de conjonction solidarisant deux implants sembleraient être des facteurs favorisants à l'aggravation de la résorption osseuse au niveau antérieur. 


\section{Les options thérapeutiques d'un édentement maxillaire complet avec une crête flottante antérieure}

Sur le plan histologique, la crête flottante présente un caractère irréversible. Elle ne disparaît pas avec la suppression de la cause, car elle ne concerne plus seulement la maturation, mais encore la différenciation cellulaire [6]. De ce fait, la mise en condition tissulaire ne peut apporter les améliorations escomptées puisque cette dernière n'agit que sur les tissus hyperhémiés [6]. Le recours au conditionneur tissulaire sera préconisé uniquement dans le cas ou la crête flottante présente une inflammation associée.

Les trois principales approches thérapeutiques curatives pour gérer cette situation clinique sont :

- l'exérèse chirurgicale de la crête flottante et la restauration de l'arcade maxillaire par prothèse amovible conventionnelle ;

- la réalisation de prothèse complète supraimplantaire maxillaire associée ou non aux greffes osseuses ;

- la réalisation de la prothèse amovible complète conventionnelle avec des techniques d'empreintes spécifiques.

Mais l'approche préventive reste le meilleur moyen pour diminuer le risque de son apparition.

\section{Exérèse chirurgicale}

\section{de la crête flottante antérieure}

En l'absence de contre indication absolue de la chirurgie, la conduite à tenir classique est la résection des crêtes flottantes. Elles sont essentiellement défavorables à la sustentation des prothèses amovibles complètes et sont donc justiciable d'une exérèse chirurgicale, chaque fois que l'état de santé du patient le permet, pour retrouver, après cicatrisation, une fibromuqueuse saine bien adhérente à I'os [19].

L'exérèse consiste en l'ablation du tissu fibreux en excès par un sondage transmuqueux sous anesthésie (pour contrôler l'épaisseur de la fibromuqueuse). Deux incisions, convergeant vers le sommet osseux, permettent l'ablation des tissus en excès (gingivectomie en quartier d'orange). Il faut éviter de supprimer trop de tissus muqueux, car aucune tension ne doit être créée au stade de la suture entre les deux lambeaux. Cela provoquerait un processus de résorption mal contrôlé du rebord alvéolaire sous-jacent. La prothèse est rebasée avec une résine à prise retardée. Un à deux mois d'attente seront nécessaire avant d'entamer les phases suivantes du traitement prothétique [20].

Malheureusement, lorsque la résorption osseuse est importante, l'exérèse chirurgicale aboutit à une surface d'appui plane avec des crêtes totalement effacées [21] et une hauteur prothétique importante au niveau antérieur. Cette morphologie assurera certes la sustentation sur un support stable, mais n'offrira aucune forme de rétention et de stabilisation. $D^{\prime}$ autant plus que la prothèse amovible complète dans ces conditions risque d'engendrer un bras de levier important au niveau antérieur, du fait que la résection aboutit à un 
espace prothétique exagéré impliquant une hauteur de résine importante pour rattraper le niveau du plan d'occlusion optimal.

Pour pallier cette problématique le recours à la prothèse supra-implantaire ou la prothèse conventionnelle avec des empreintes à pression sélective s'avèrent des alternatives séduisantes.

\section{Gestion par prothèse supra-implantaire maxillaire}

La prothèse complète fixée transvissée, la prothèse fixée amovo-inamovible et la prothèse à complément de rétention implantaire, offrent les moyens les plus efficaces pour améliorer la stabilité, la rétention et la fonction d'une prothèse complète.

En présence de crête flottante antérieure maxillaire, I'os sous jacent est atrophié. Le placement d'implants courts dans un os faiblement dense et richement vascularisé augmente le risque d'echec [22, 23]. Engquist et al. [24] ont noté que le taux d'échec est multiplié par un facteur de 2 à 3, lorsque des implants courts de 7 à 10 $\mathrm{mm}$ sont placés au niveau maxillaire. De même, l'échec implantaire est multiplié par 2 à 3 lorsque le volume ou la qualité osseuse sont évalués comme les plus faibles.

Ainsi,quand la hauteur occluso-prothétique utilisable antérieure est importante, plusieurs techniques chirurgicales spécifiques permettent l'augmentation de la hauteur de l'os de la crête antérieure [23]. Parmi elles citons :

- les greffes d'os autogènes d'origine iliaque $[24,25]$;

Keller et al. [26] ont rapporté un taux d'échec de $23 \%$ pour les greffes d'os autogènes en blocs apposés " onlay block » servant à traiter les résorptions maxillaires importantes en complément de PACSI. Ces auteurs ont également montré que la conception prothé- tique fixée maxillaire associée à un contrôle des forces occlusales transmises améliore les résultats des greffes d'apposition dite en onlay.

- l'épaississement de la crête maxillaire à l'aide d'un lambeau d'os du péroné vascularisé libre, préfabriqué, associé à des implants [27] ;

- la distraction alvéolaire verticale ostéogène [28] ; et l'expansion de la crête avec la pose immédiate d'implants [30] ;

- l'utilisation de greffons osseux et d'implants transitoires [29].

Afin d'éviter les procédures chirurgicales compliquées et couteuses des greffes osseuses, le placement d'implants au niveau des secteurs latéraux associés aux implants angulés dans des sites postérieurs est proposé [31, 32]. Aparicio et al. [33] ont rapporté que la perte osseuse marginale autour d'implants angulés à $15^{\circ}$ et d'implant axiaux n'est pas différente après sept années de suivi. Le placement d'implants zygomatiques $[34,35]$ ou ptérygomaxillaires $[36,37]$ procure un résultat satisfaisant dans le traitement des crêtes maxillaires résorbées traitées par prothèses complètes fixées supra-implantaire.

La restauration par prothèse amovible supraimplantaire avec des ancrages zygomatiques et deux implants antérieurs présente des contours volumineux en regard des implants postérieurs ; cela nécessite le recours à des piliers antérieurs angulés et/ou à une position linguale de la barre de conjonction.

Tolstunov [38] propose une réhabilitation maxillaire par une prothèse complète supraimplantaire en gardant la crête flottante antérieure. La prothèse est connectée par deux barres de conjonctions séparées droite et gauche, avec trois implants au niveau de chaque secteur postérieur. Cette répartition des sites implantaires semble être plus favorable sur 
le plan biomécanique en évitant la concentration des forces dans les régions antérieures.

La restauration par prothèses supra-implantaire maxillaire, d'une part, change la distribution des forces occlusales et favorise la transmission des stimuli fonctionnels à l'os péri implantaire. Ceci est un facteur qui limite considérablement la résorption osseuse [39]. Mais d'autre part, ce type de restauration présente un taux d'échec relativement élevé, une durée de traitement longue, des temps chirurgicaux qui peuvent être lourds, de même, qu'un coût initial et de maintenance considérablement élevé $[40,41]$.

\section{Gestion par prothèse amovible complète conventionnelle avec des techniques d'empreintes spécifiques}

Elle consiste à réaliser des empreintes réduisant le potentiel de déplacement des crêtes flottantes lors de la fonction.

L'empreinte primaire doit être mucostatique au plâtre ou à l'alginate à consistance fluide limitant au maximum l'écrasement et la compression de la fibromuqueuse mobile [42].

L'empreinte secondaire doit enregistrer la crête flottante à l'état mucostatique, sans la comprimer ni la déplacer, alors qu'elle doit être anatomofonctionnelle et assurer une légère compression au niveau du reste de la surface d'appui saine dans la limite de l'homéostasie de la muqueuse : c'est une empreinte mixte à pression sélective [43].

La gestion de l'empreinte se fait à deux niveaux:

- la confection d'un porte empreinte individuel adapté mais non compressif en regard du tissu fibreux antérieur par:

- la décharge du PEI (porte-empreinte individuel) à ce niveau et la création de perforation permettant l'échappement du maté- riau à empreinte [44]. Ce type de porteempreinte sera indiqué quand la hauteur de la crête flottante est faible avec absence de contre-dépouille au niveau antérieur pour permettre la remise en place du PEI lors des différentes manœuvres ;

- par la fenestration du PEI sur le pourtour de la crête flottante avec possibilité d'enregistrement anatomofonctionnel du joint périphérique vestibulaire antérieur [3, 45]. Cette option est préconisée en présence d'une hauteur importante du tissu fibreux. Certains auteurs préconisent même un PEI complètement ouvert au niveau antérieur $[44,46,47]$, ou composé de deux parties [47], une partie servant à soutenir la crête flottante du côté palatin et une partie servant à réaliser une empreinte globale. Ces techniques sont réservées aux crêtes flottantes de grande hauteur avec une contre dépouille antérieure importante ;

- le choix des matériaux à empreinte pour minimiser le déplacement du tissu mou, la majorité des techniques utilise des matériaux de faible viscosité (Plâtre de Paris, élastomère de faible viscosité) [19, 42].

Les deux techniques d'empreinte à pression sélective les plus utilisées, sont illustrées ciaprès :

- une technique avec PEI fenestré en deux temps :

la patiente présente un édentement maxillaire en face d'une classe II Kennedy Applegate. La surcharge occlusale au niveau antérieur a généré une résorption osseuse accompagnée d'une crête flottante à ce niveau. Face au refus catégorique de la patiente pour la chirurgie et vu que l'espace prothétique utilisable est suffisant, la prothèse conventionnelle avec une empreinte sélective est proposée. 
Après une estimation clinique de l'étendue et de l'épaisseur de la fibromuqueuse non adhérente (fig. 1) la réalisation d'une empreinte anatomo-fonctionnelle est conduite de façon classique en réalisant un remarginage suivi d'une empreinte de surfaçage (fig. 2 a et $\mathbf{b}$ ). Puis, le porte-empreinte est fenestré en regard de la crête flottante en préservant le joint périphérique (fig. 3). Le PE (porte-empreinte) est validé cliniquement, la totalité de l'hyperplasie est dégagée en évitant toute compression ou déplacement (fig. 4). Le matériau de faible viscosité est injecté (polysulfure) et l'empreinte est désinserée après sa prise (fig. 5, fig. 6).

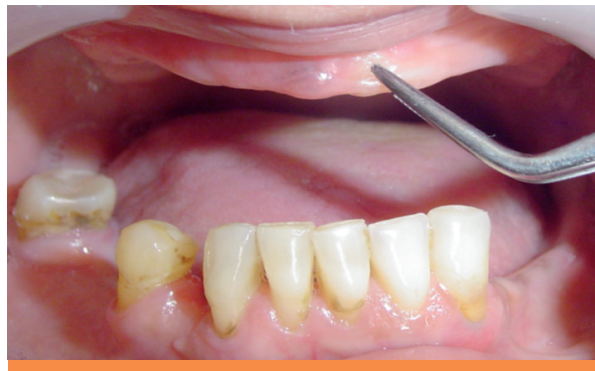

Fig. 1 Estimation clinique de l'étendue et de l'épaisseur de la fibromuqueuse non adhérente.
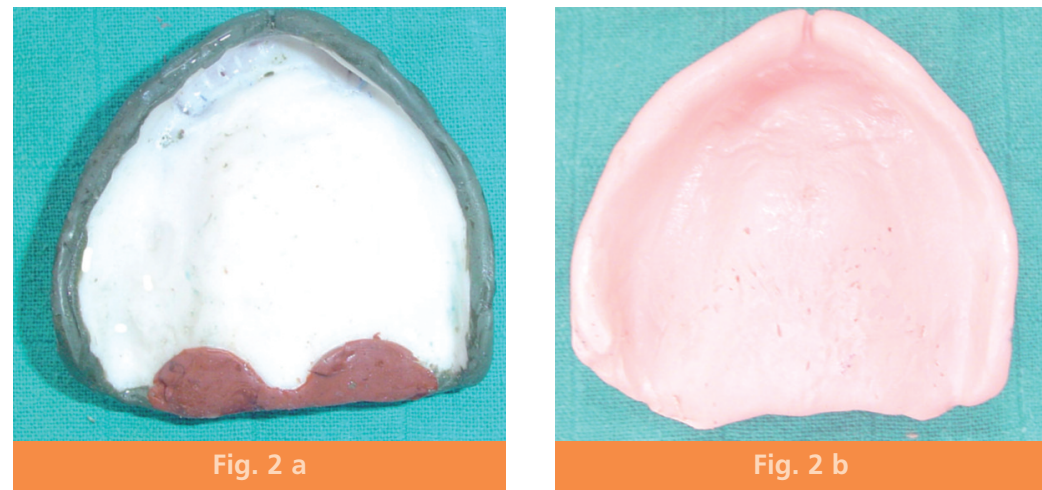

Fig. 2 a et b Réalisation d'une empreinte anatomo-fonctionnelle classique.
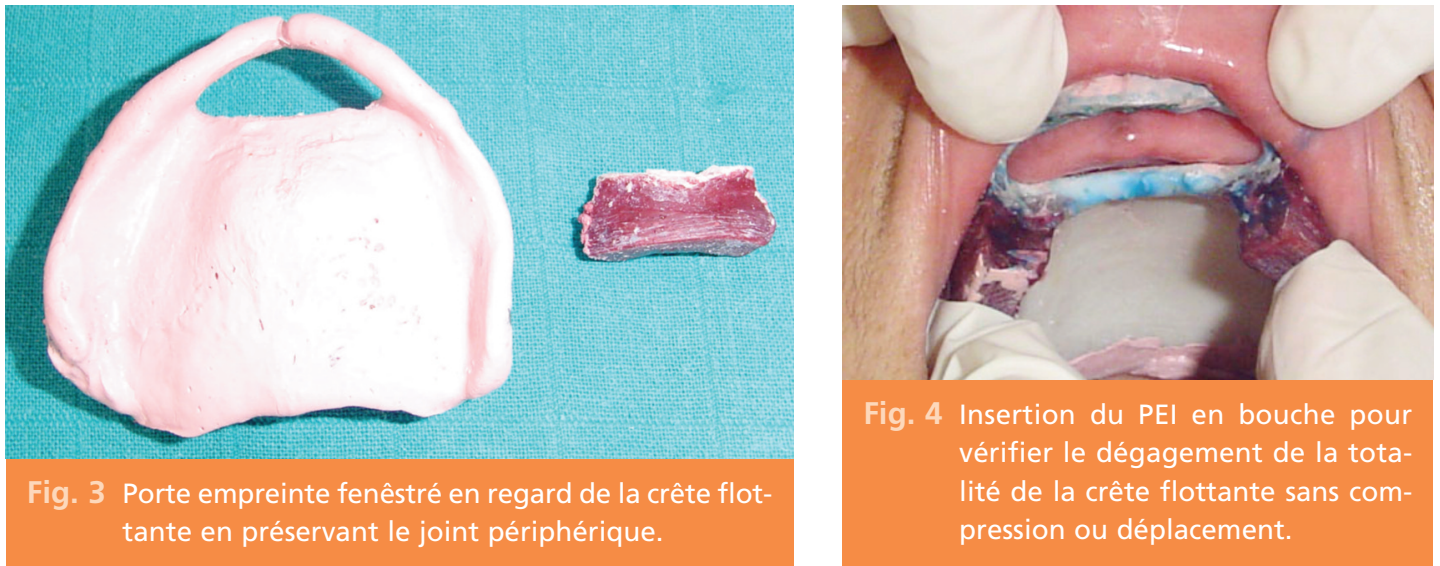

Fig. 4 Insertion du PEI en bouche pour vérifier le dégagement de la totalité de la crête flottante sans compression ou déplacement.
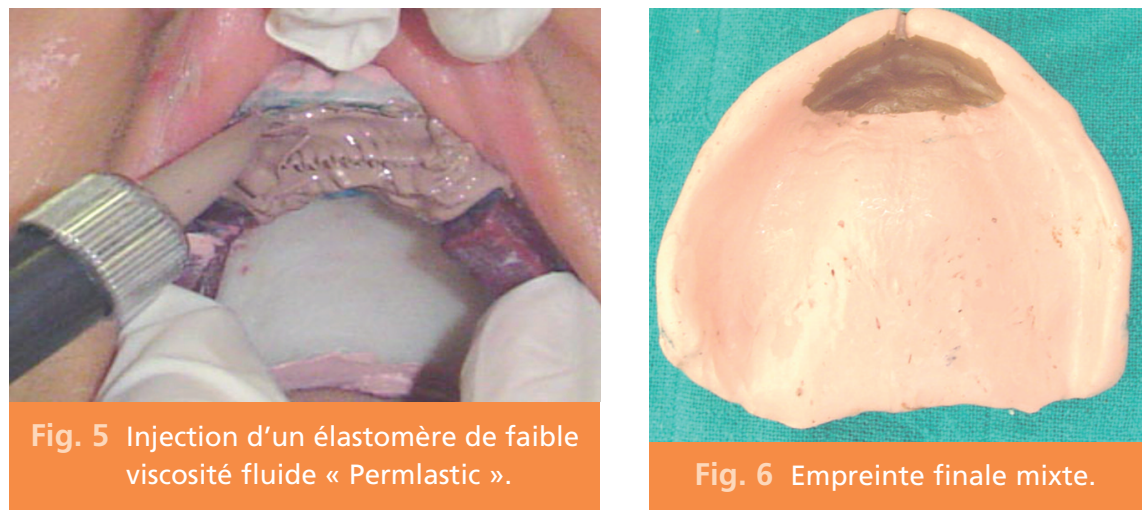
- une technique avec PEI déchargé en deux temps :

la patiente est porteuse d'une prothèse amovible complète bimaxillaire. L'examen détecte un proglissement mandibulaire avec un articulé en bout à bout antérieur (fig. 7). Il s'avère que la patiente durant sept ans n'a jamais effectué de contrôle et

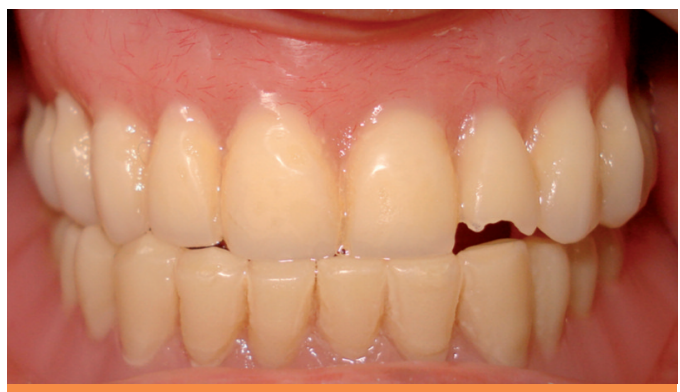

Fig. 7 Vue endobuccale des prothèses en occlusion.
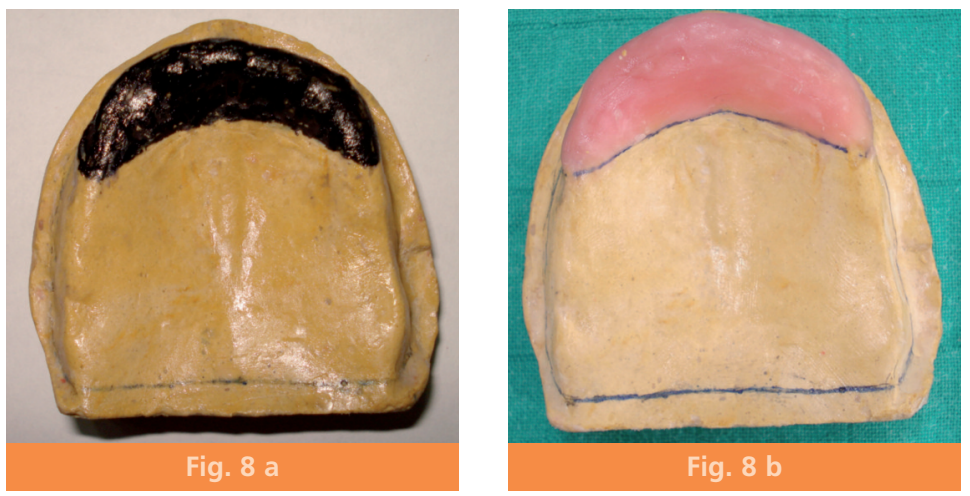

Fig. 8 a et b Réalisation d'un PEl espacé en regard de la crête flottante antérieure par une épaisseur de cire..

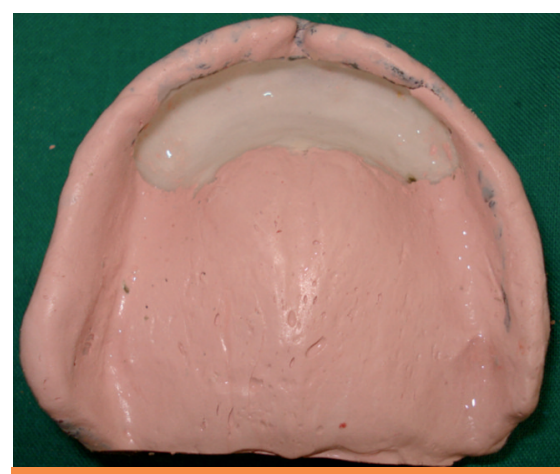

Fig. 9 Réalisation de l'empreinte anatomo-fonctionnelle classique et élimination à l'aide d'un bistouri du matériau de surfaçage en regard de la crête flottante.

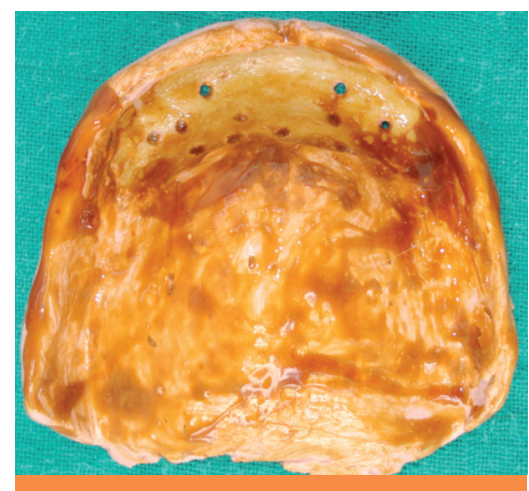

Fig. 10 Application d'adhésif pour élastomère et perforation du PEI au niveau de la zone antérieure déchargée.

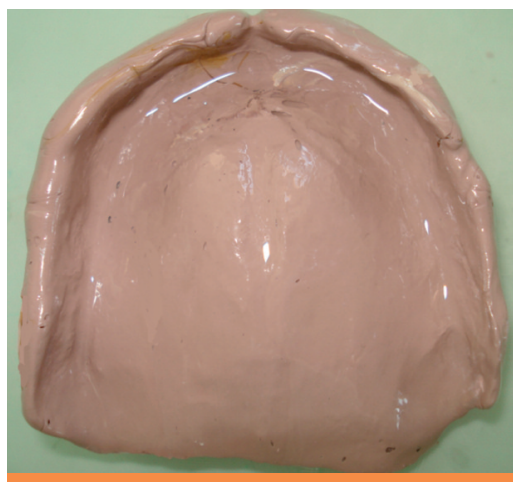

Fig. 11 Empreinte finale mixte. 
que la résorption osseuse n'a pas été compensée. Le contact antérieur entre les prothèses complètes a généré une surcharge de la crête maxillaire correspondante.

La patiente est porteuse d'une prothèse valvulaire ce qui complique l'indication de I'acte chirurgical. D'où l'orientation vers la réalisation de nouvelle prothèse associée à une empreinte mixte.

Un PEI est confectionné avec un espacement d'une épaisseur de cire en regard de la crête flottante, délimitée sur le modèle primaire, (fig. 8 a et b). Une fois l'empreinte anatomofonctionnelle classique réalisée, la zone en regard de la crête flottante est déchargée (fig. 9) et perforée (fig. 10). L'ensemble de l'empreinte est ensuite surfacé par un élastomère de faible viscosité (fig. 11).

Sachant que la plupart des patients édentés totaux sont âgés, la prothèse complète conventionnelle associée aux techniques d'empreintes sélectives est une solution intéressante.
En effet, les patients âgés sont généralement demandeurs, de sécurité, de rapidité, d'économie, de traitement sans douleur. Ils présentent trois types de difficultés [48] :

- obstacle d'ordre physique : la plupart présentent des pathologies multiples et prennent régulièrement plusieurs médicaments par jour, ce qui peut compliquer l'indication des thérapeutiques chirurgicales ;

- d'ordre psychique : un certain nombre de signes seront également associés au grand âge et à la sénilité. Parmi ceux-ci citons les dépressions, l'impuissance, l'indécision, l'anxiété... ce qui les rend moins favorables aux traitements complexes et moins ouverts aux nouvelles techniques (notamment l'implantologie) ;

- et d'ordre économique : la plupart sont des retraités connaissant des problèmes de couverture sociale et des moyens financiers souvent limités, ce qui implique des thérapeutiques moins onéreuses.

\section{La thérapeutique préventive}

La prévention de l'apparition des crêtes flottantes maxillaires consiste à :

- préserver les racines dentaires en les exploitant comme moyen d'ancrages prothétiques radiculaires ; cela permettra de maintenir le capital osseux et la proprioception parodontale ;

- réaliser des extractions atraumatiques ou parodontales quand l'indication est posée pour diminuer la résorption osseuse primaire ;

- adopter un protocole rigoureux lors de la réalisation prothétique pour éviter les erreurs de conception favorisant l'apparition de contraintes excessives ;
- éviter les restaurations ponctuelles et favoriser les réhabilitations globales bimaxillaires assurant des contacts occlusaux postérieurs, évitant la surcharge de la crête antérieure et restaurant une occlusion bilatéralement équilibrée ;

- préférer, quand cela est possible, les prothèses fixées mandibulaires par rapport aux autres conceptions amovibles ;

- établir une planification des visites périodiques de contrôle pour dépister les signes précurseurs de la résorption de la crête antérieure maxillaire. 


\section{Conclusion}

L'élimination chirurgicale des crêtes flottantes était souvent préconisée, car celles-ci peuvent être à l'origine d'une instabilité prothétique et limitent fortement la transcription des forces occlusales au substratum osseux sousjacent. Cependant, actuellement, les auteurs conseillent de gérer cette situation par des techniques conservatrices, en utilisant des empreintes spécifiques ou par des reconstructions prothétiques supra-implantaires [3].

La gestion par prothèse complète conventionnelle est une thérapeutique conservatrice préservant l'intégrité tissulaire, peu couteuse.

Elle offre un résultat sur le plan biomécanique très modeste mais qui $n$ 'implique pas la réalisation d'une prothèse insatisfaisante.
Les thérapeutiques implanto-supportées ne cessent d'évoluer. Elles procurent des prothèses très stables et un maintien du capital osseux durable dans le temps.

Mais elles nécessitent des interventions chirurgicales, parfois complexes, un temps de traitement parfois long et un investissement financier souvent important pour les patients.

La démarche décisionnelle prothétique doit tenir compte de la notion du rapport bénéfice/risque, particulièrement pour la majorité de ces patients édentés complets qui présentent souvent un contexte psychologique, physique et socio-économique fragiles.

\section{Bibliographie}

1. Le Bars $\mathrm{P}$, et al.

Réactions tissulaires au port des appareils de prothèse dentaire amovible partielle ou totale.

EMC Odontologie édition 2002[23-325-P-10].

2. Carlsson GE. Responses of jawbone to pressure.

Gerodontology 2004;21:65.

3. Hüe $O$, Berteretche MV. Prothèse complète : réalité clinique, solutions thérapeutiques. Quintessence International, 2004.

4. Kelly E. Changes caused by a mandibular removable partial denture opposing a maxillary complete denture.

J Prosthet Dent 2003;90:213-9.

5. Magnusson BC, Engstrom $\mathrm{H}$, Kahnberg K-E. Metaplasic formation of bone and chondroid in flabby ridge. $\mathrm{Br} J$ Oral Maxillofac Surg 1986;24:300-305.

6. Ogolnik R. Édentation totale et chirurgie préprothétique : chirurgie des parties molles. Cah Proth 1981;34:55-60.

7. Kelly E.

Changes caused by a mandibular removable partial denture opposing a maxillary complete denture.

J Prosthet Dent 1972;27:140150.

8. Xie Q, Ainamo A, Tilvis $R$. Association of residual ridge resorption with systemic factors in the home living elderly subjects.

Acta Odontol Scand 1997;55:299-305

9. Zarb GA, Bolender C, Carlsson GE.

Boucher's prosthodontic treatment for edentulous patients.

St Louis: CV Mosby, 1997

10. Lynch CD, Allen PF.

Case report:

Management 
of the flabby ridge: Re-visiting the principles of complete denture construction.

Eur J Prosthodont Restor

Dent 2003 Dec;11(4):145-8.

11. The glossary of prosthodontic terms. J Prosthet Dent 1999;81:39-110.

12. Kelly E.

Changes caused by a mandibular removable partial denture opposing a maxillary complete denture.

J Prosthet Dent 1972;27:140-150.

13. Saunders TR, Gillis RE Jr, Desjardins RP. The maxillary complete denture opposing the mandibular bilateral distal-extension partial denture: treatment considerations. J Prosthet Dent 1979 Feb;41(2):124-8.

14. Palmqvist $\mathrm{S}$, Carlsson GE, Öwall B.

The combination syndrome: A literature review. J Prosthet Dent 2003;90:270-275.

15. Narhi TO, Geertman ME, Hevinga $M$, et al.

Changes in the edentulous maxilla in persons wearing implant-retained mandibular overdentures. J Prosthet Dent 2000;84(1):43-49.

16. Abd El-Dayem MA, Assad AS, Abdel-Ghany MM. The effect of different mandibular dentures on antagonistic maxillary ridge. Implant Dent 2007 Dec;16(4):421-9.
17. Kreisler M. et al. Residual ridge resorption in the edentulous maxilla in patients with implantsupported mandibular over dentures: An 8-year retrospective study. Int J Prosthodont 2003 May-Jun;16(3):295-300.

18. Gupta S, Lechner SK, Duckmanton NA. Maxillary changes under complete dentures opposing mandibular implant-supported fixed prostheses. Int J Prosthodont 1999;12:492.

19. Rignon-Bret $C$, Ringon-Bret JM.

Prothèse amovible complète, prothèse immédiate, prothèse supraradiculaire et implantaire.

Paris: Éditions CdP, Collection JPIO; 2002:39.

20. Castagny $E$, Laffargue $P$, Godet C.

Aménagement préprothétique des crêtes édentées : six situations cliniques fréquentes. Inf Dent $n^{\circ} 38$, nov 2002.

21. Owall B, Kayser AF, Carlsson GE.

Prothèse dentaire : principes et stratégies thérapeutiques.

Paris: Elsevier Masson, 1998.

22. Goodacre CJ ,Kan JYK, Rungchrassaeng K. Clinical complication of osseointegrated implants. J Prosthet 1999;81:531-552.

23. Watson RRM et al. Prosthodontic treatment, patient response and the need of maintenance of complete supported overdentures.
An appraisal of five years of prospective study. Int J Prosthodont 1997; 10:345-354.

24. Engquist $B$, Bergendal $T$, Kallus $\mathrm{T}$, Linden $\mathrm{U}$.

A retrospective multicenter evaluation of osseointegrated implants supporting overdentures. Int J Oral Maxillofac Implants, 1988; 3:129-34.

25. Maiorana $C$, Santoro $F$, Rabagliati M, et al. Evaluation of the use of iliac cancellous bone and anorganic bovine bone in the reconstruction of the atrophic maxilla with titanium mesh: A clinical and histologic investigation.

Int J Oral Maxillofac Implants, 16:427,2001.

26. Keller EE, Tolman DE, Eckert $\mathrm{S}$.

Surgicalprosthodontic reconstruction of advanced maxillary bone compromise with autogenous onlay block bone grafts and osseointegrated endosseous implants: a 12-year study of 32 consecutive patients. Int J OralMaxillofac Implants, 1999; 14:197-209.

27. Rohner D, Bucher $P$,

Kunz C, et al.

Treatment of severe atrophy of the maxilla with the prefabricated free vascularized fibula flap. Clin Oral Implants Res 13:44,2002.

28. Yalcin $S$, Ordulu $M$, Emes $Y$, et al. Alveolar distraction osteogenesis before placement of dental implants. Implant Dent 15:48,2006. 
29. Petrungaro PS. Reconstruction of severely resorbed atrophic maxillae and management with transitional implants. Implant Dent 9:271,2000.

30. Guirado JL, Yuguero MR, Carrion del Valle MJ, et al. A maxillary ridge-splitting technique followed by immediate placement of implants: A case report. Implant Dent 14:14,2005.

31. Calandriello $\mathrm{R}$, Tomatis $\mathrm{M}$. Simplified treatment of the atrophic posterior maxilla via immediate/early function and tilted implants: A prospective 1-year clinical study.

Clin Implant Dent Relat Res 2005;7 Suppl 1:S1-12.

32. Krekmanov L, Kahn M, Rangert B, Lindstrom $\mathrm{H}$. Tilting of posterior mandibular and maxillary implants for improved prosthesis support. Int J Oral Maxillofac Implants, 2000;15:405-14.

33. Aparicio $C$, Perales $P$, Rangert B.

Tilted implants as an alternative to maxillary sinus grafting: a clinical, radiologic, and periotest study. Clin Implant Dent Relat Res 2001;3:39-49.

34. Malevez C, Abarca M, Durdu F, Daelemans P. Clinical outcome of 103 consecutive zygomatic implants: a 6-48 months follow-up study. Clin Oral Implant Res 2004;15:18-22.

35. Aparicio C, Ouazzani W, Garcia R, Arevalo X, Muela R, Fortes V. A prospective clinical study on titanium implants in the zygomatic arch for prosthetic rehabilitation of the atrophic edentulous maxilla with a follow-up of 6 months to 5 years. Clin Implant Dent Relat Res 2006;8:114-22.

36. Balshi TJ, Wolfinger GJ, Balshi SF.

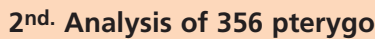
maxillary implants in edentulous arches for fixed prosthesis anchorage.

Int J Oral Maxillofac, Implants 1999;14:398-406.

37. Worthington $P$, Branemark PI.

Advanced osseointegration surgery:

Maxillofacial applications.

Chicago: Quintessence, 1992:182-8.

38. Tolstunov $\mathrm{L}$. Management of Biomechanical Complication of Implant-Supported Restoration of a patient with Combination Syndrome: A case report.

J Oral Maxillofac Surg 67:178-188, 2009.

39. Carlsson GE. Responses of jawbone to pressure. Gerodontology 21:65,2004.

40. Watson RRM, Jemt $\mathrm{T}$,

Chai J, et al.

Prosthodontic treatement patient response and the neef for maintenance of complete supported overdentures: An appraisal of five years of prospective study. Int J Prosthodontic 1997;10:345-354.

41. Dunnen $A C L$, et al. Adjusment and complication of mandibular overdentures retained by four implants.

A comparison between superstructures with and without cantilever extensions. Int J Prosthodont 1998;11:307-311.

42. Lynch $C D$, Allen PF. Management of the flabby ridges: Using contemporary materials to solve an old problem. Br Dent J. 2006 Mar 11; 200(5):258-261.

43. Lamb DJ.

Problems and solutions in complete denture prosthetics.

London: Quintessence publishing ; 1993:57-60.

44. Langer $\mathrm{Y}$, et al. Modalities of treatment for the combination syndrome. J Prosthod 1995;4:76-81.

45. Allen PF, McCarthy S. Complete dentures: from planning to problem solving. London: Quintessence publishing; 2003:48-51.

46. Lynch $C D$, Allen PF. Case report: management of the flabby ridge: re-visiting the principles of complete denture construction.

Eur J Prosthodont Restor Dent 2003 Dec;11(4):145-8.

47. Allen $F$.

Management of the flabby ridge in complete denture construction. Dent Update 2005 Nov;32(9): 524-6,528.

48. Sacy A, El Hage F. Plans de traitement chez les personnes âgées. Les Cahiers de l'Adf $n^{\circ} 8-2000$. 


\section{SUMMARY}

\section{Management of edentulous maxillae with an anterior flabby ridge}

Jihane SLAOUI HASNAOUI, Amal SEFRIOUI, Olivier FROMENTIN, Ahmed ABDEDINE

\section{Keywords}

- flabby ridges - edentulous maxillae - complete dentures - maxillary resorption
The flabby ridges are present in $24 \%$ of edentulous maxillae with a preference for the anterior region; they are often associated with the combined syndrome described by Kelly.

On the histological plan, the flabby ridge presents an irreversible character. Therefore, the use of tissue conditioner material cannot bring the expected improvements.

Three main curative therapeutic approaches to manage this clinical situation are:

- surgical removal of fibrous tissue and the restoration with conventional complete denture;

- implant retained prosthesis partner or not in bone grafts;

- conventional complete removable prosthesis with specific impressions techniques.

The purpose of this article is to make the synthesis of these various available therapeutic possibilities and to discuss their indications.

\section{Abrégé de psychologie à l'usage des praticiens \\ " Lire entre les dents »}

\section{M.G. Choukroun}

Cet ouvrage est destiné à former les praticiens sur un champ de connaissances beaucoup trop négligé dans notre profession de santé. En effet, la psychologie est un domaine complexe et seul un odontologiste ayant la double formation pouvait se permettre d'en concevoir les applications spécifiques.

Conçu pièce par pièce le lecteur pourra aborder cet ouvrage au hasard des chapitres qui attirent sa curiosité, mais en se prenant au jeu de la lecture, il pourra se construire une connaissance complète des bases fondamentales en ce qui concerne la coopération thérapeutique.

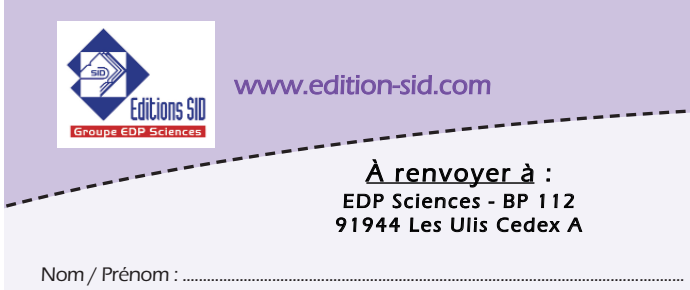

Adresse :

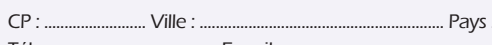

Tél. : E-mail :
232 pages, noir et blanc format $17 \times 24 \mathrm{~cm}$ $68 € \pi T C$
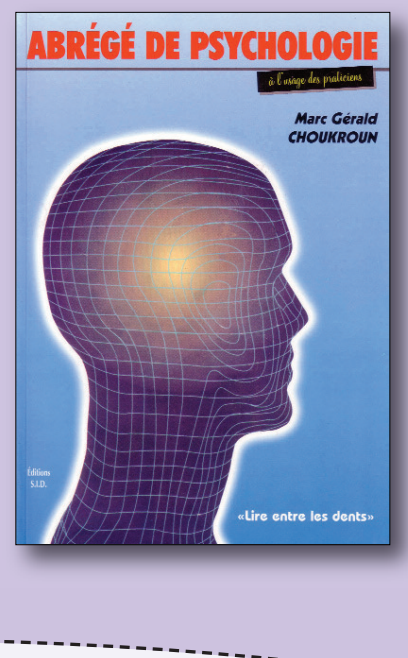

Prix unitaire Quantité Tota

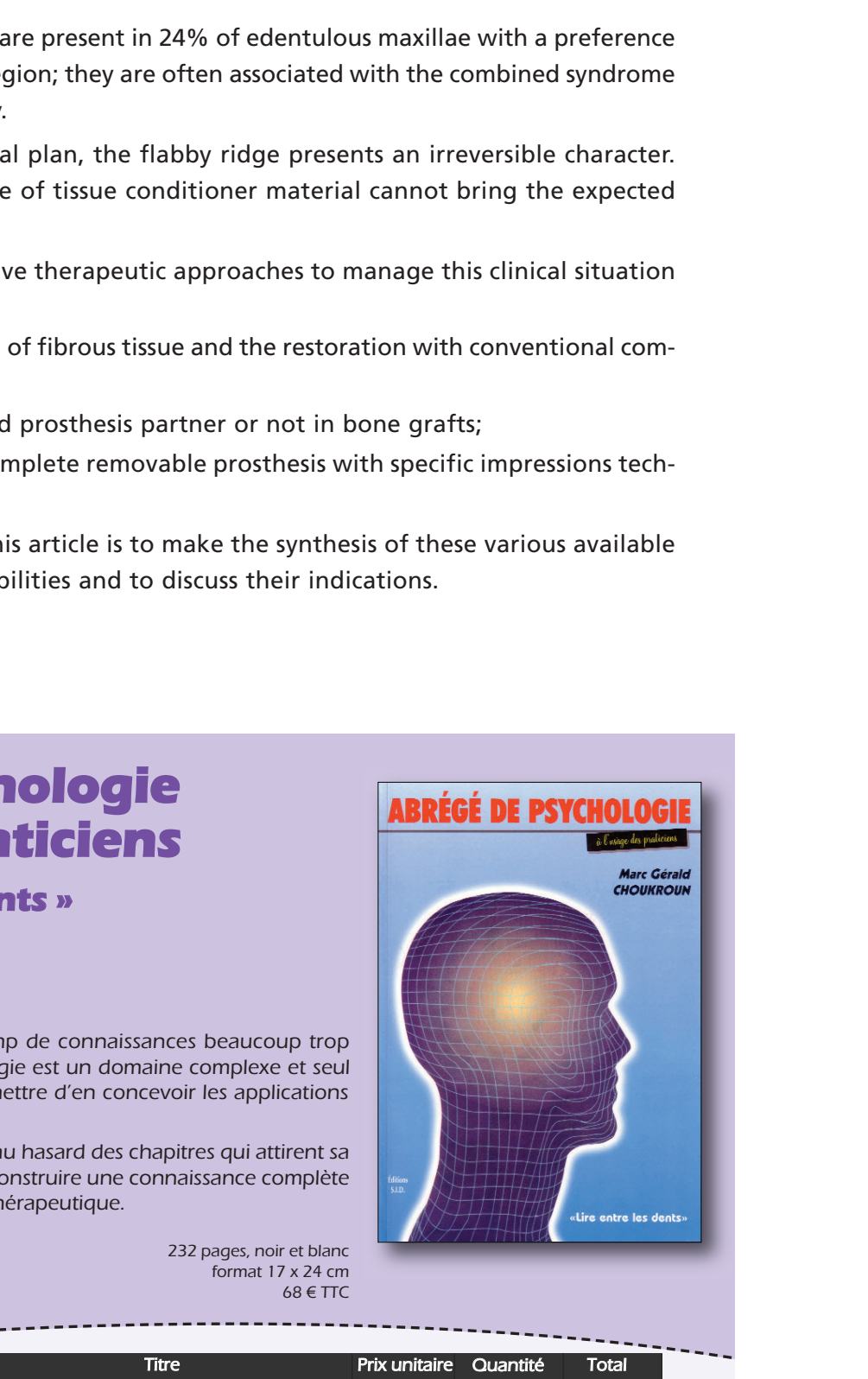

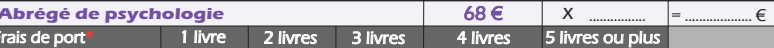

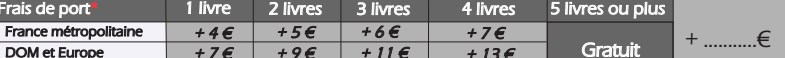

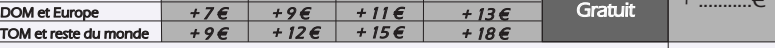
"Aucune commande ne pourra être expédiée sans ajout des frais de port. $\quad$ TOTAL GÉNÉRAL $=\ldots . . . . . . . . . . . . . \epsilon$ $\stackrel{0}{N}$
ù
o

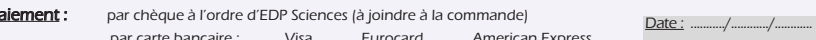
$N^{*}$ de carte $\downarrow+|+|+\mid$ Date d'expiration :................. 